\title{
An IBM XT-compatible, computer-based, slide-projector laboratory
}

\author{
PHILIP K. STODDARD and GEOFFREY R. LOFTUS \\ University of Washington, Seattle, Washington
}

\begin{abstract}
In the present paper, we describe the software and hardware of an on-line, visual-memory laboratory running under Turbo Pascal on an IBM PC XT-compatible computer. The display system includes one Kodak random access projector and four standard Kodak carousel projectors, all equipped with tachistoscopic shutters and luminance-control devices. The response system consists of eight 16-key response boxes. The laboratory can be used for any experiment in which 35-mm slides are to be used as stimuli, and in which precise display times, stimulus luminances, and reaction times are required. The laboratory is particularly well suited to picture-perception and picture-memory experiments.
\end{abstract}

Loftus, Gillispie, Tigre and Nelson (1984) describe an Apple II-based laboratory system designed for performing experiments that involve image presentation and subject response. The system allows tachistoscopic stimulus presentation from four 35-mm slide projectors; control of stimulus luminance via neutral-density filters; vocal and digital reaction times; and on-line response collection (including reaction times) from up to 8 subjects at once. This laboratory has been used to collect data from approximately 7,500 subjects, who participated in approximately 100 experiments (a sampling of these experiments is described in Loftus, 1985a, 1985b; Loftus \& Ginn, 1984; Loftus, Hanna, \& Lester, 1988; Loftus \& Hogden, 1988; Loftus, Johnson, \& Shimamura, 1985; Loftus, Truax, \& Nelson, 1986; and Reinitz, 1987).

We describe here a successor to this laboratory. Our goal in constructing it was to correct problems in the original prototype. The new laboratory was to run under the reasonably fast and very easy-to-use Turbo Pascal (Version 4.0) on an MS-DOS-based, IBM XT-compatible computer. Second, the display system needed to be completely soundproof from the subjects' perspectives, the projectors within needed to be easily accessible for alignment and servicing, and the system had to generate less equipment-damaging heat than did its predecessor. Third, we wanted to incorporate five separate projectors that were arranged closely enough together to be mutually aligned, thereby avoiding parallax problems, yet with enough space to provide a luminance-control device for each projector. Fourth, we wanted to eliminate electrical noise that had caused occasional spurious shutter activity in the original laboratory. Fifth, we wanted to be able to distinguish successive subject responses on the same key, a feature that had been omitted from the original system.

The building of this laboratory was supported by an NIMH grant to Geoffrey Loftus. We thank Mike Burdette and Greg Gallucci for technical assistance. Walter Taucher integrated the IBM XT-compatible computer. Correspondence may be addressed to Geoffrey R. Loftus, Department of Psychology, University of Washington, Seattle, WA 98195.

\section{SYSTEM OVERVIEW}

Figure 1 shows the gross configuration of the laboratory. The display system (projectors, shutters, and neutraldensity filters) is housed in a $4 \times 4 \times 5 \mathrm{ft}$ box. Up to 8 subjects sit in two four-chair rows, with the back row on a platform raised $2 \mathrm{in}$. higher than the front. The subjects look at a wall covered entirely with black velvet apart from a $2 \times 3 \mathrm{ft}$ white display screen (the velvet is necessary to absorb spurious reflections from the various optical components of the display system). The visual angle subtended by a displayed slide ranges from $20^{\circ}$ to $31^{\circ}$, depending on where the observer is seated.

The laboratory is controlled by an IBM XT-compatible 8088-based computer with $640 \mathrm{~K}$ of memory (our software will run on any IBM-compatible computer with at least 384K). The only nonstandard computer hardware consists of three Data Translation DT2817 parallel I/O cards, a QuaTech PBX-721 expansion card (three Intel 8255 PPI chips), a QuaTech CTM-10 plug-in timing module (Intel 8253 counter/timer chip with on-board oscillator) that increments a 16-bit register at 1-ms intervals, and a custom-built board to detect the strobed signals indicating keypresses at the response boxes. The computer uses the standard MS-DOS (Version 3.2) operating system, and all laboratory software is written in Turbo Pascal (Version 4.0). All components of both the display and response systems can be accessed via procedures and functions called by Pascal user programs.

The documentation for the interface boards from QuaTech Inc. was inadequate; it had to be supplemented with schematic diagrams from the company, and with the microprocessor manuals from Intel. QuaTech now sells a subroutine library for Turbo Pascal. Another problem is that noise on the bus of many IBM compatibles (such as ours) resets the parallel expansion board's 8255 PPI chips at random intervals. We are informed that this problem can be safely remedied by clipping the reset line to the 8255 chip (pin 35 ). The 8255 chip on our board 


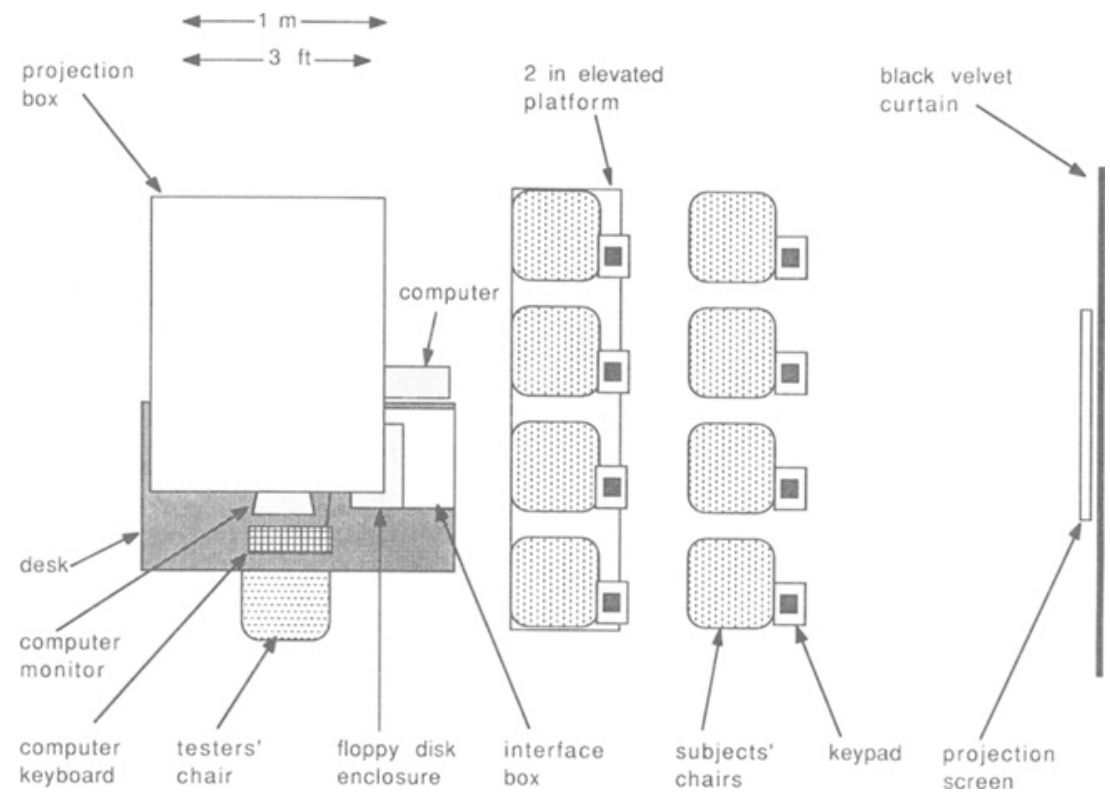

Figure 1. Schematic of room.

has needed replacement three times in one year. There must be a more reliable timer board available.

The display system consists of four standard Kodak Ectagraphic IIIA projectors (Projectors 1-4) and one Kodak random access projector (Projector 5). Each is equipped with a Gerbrands tachistoscopic shutter and a luminance-attenuation device (described below). The standard IBM PC tone generator is software-integrated with the display apparatus.

\section{THE DISPLAY SYSTEM BOX}

The basic hardware configuration within the projection box is shown in Figures 2 and 3. Figure 2 shows a side view from the operator's perspective, and Figure 3 shows a front view. The box consists of three major components: the shell, the soundproof cooling system, and the equipment rack. The shell was designed to contain the broad band clicks generated by the tachistoscopic shutters and the much louder projector advance mechanisms. This was essential so that subjects would receive no clues as to the nature of stimuli being prepared for the next presentation. The shell is made of four airtight layers. Starting from the outside of the box and moving inward, they are: 1-in. thick Kortron high-density particle board finished on both sides, 3/8-in. closed-cell foam, 1/2-in. drywall plasterboard, and 4-in. Sonex acoustic foam. The projected images pass through three sealed panes of laminated glass. The inset door seals firmly against an internal flange. Sound isolation is so complete that only by pressing one's ear firmly against the outside of the shell is it possible to detect the advance of the projectors.

To remove projector heat, an ABS plastic tube mates with the cooling fan exhaust vent of each projector. Each tube runs into a common column. The column vents to a labyrinth duct system in the back of the box. The ductwork is made of drywall, lined with Sonex 1 acoustic absorption material. Air passing through the labyrinth makes eight $90^{\circ}$ turns before exiting the box and passing into the building ventilation system. An identical labyrinth prevents sound from escaping the air intake duct as well. The box is positively pressurized by fans moving room air at $250 \mathrm{ft}^{3} / \mathrm{min}$.

Inside the box is a steel rack with five sliding drawers, one for each projector assembly. The sliding drawers facilitate close stacking of the projectors but still allow experimenters to remove slide trays and service the projec-

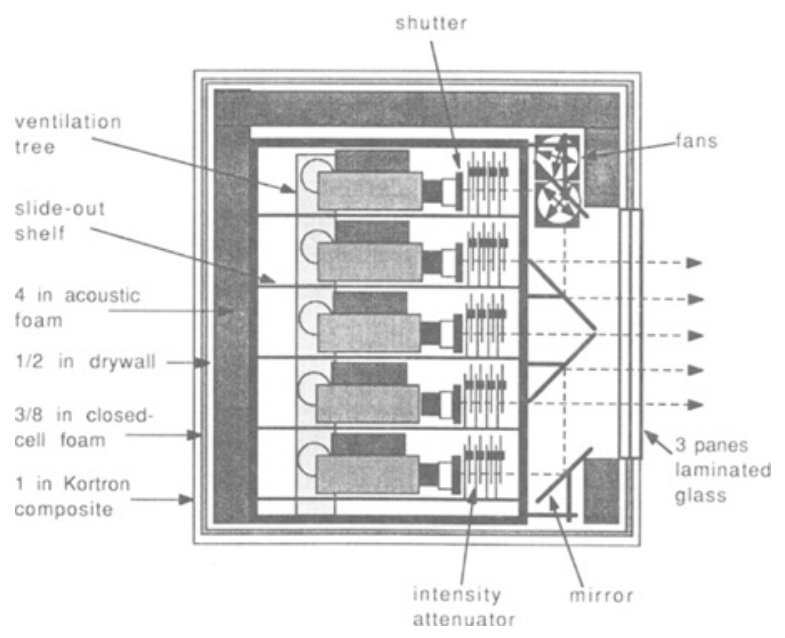

Figure 2. Schematic of the projection box viewed from the access side, with the door and door seal removed to reveal a cross section of the acoustic damping structures. 


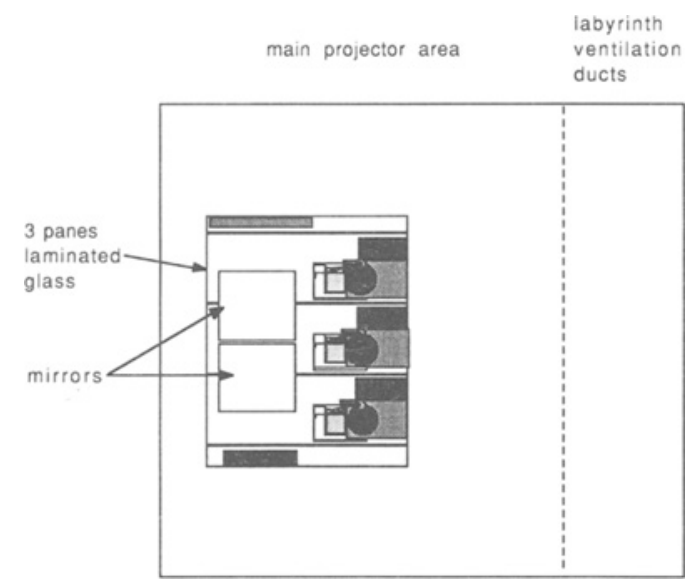

Figure 3. Schematic of the projection box as viewed by a subject looking backward from the screen.

tors. All electrical connections within the box are made with high quality gold-plated connectors for reliability, with an emphasis on modularity and ease of removal. The images projected from the top and bottom projectors are reflected periscopically off front surface mirrors on adjustable mounts, so that their images exit the box at about the same elevation as the middle three projectors. Zoom projection lenses are used to correct image sizes for the different path lengths. Without the periscopes, images aligned from the upper and lowermost projectors would appear on the screen as distinct trapezoids instead of rectangles. A feathered Mardi-Gras mask hangs on the outside of the box to ward off evil spirits.

\section{SYSTEM HARDWARE AND SOFTWARE}

Each of the five projectors has a shutter and a luminance-control device. The four standard projectors have advance and reverse capabilities. The solid-state relay modules and ramped shutter drivers are placed in a separate, electrically shielded box that connects with the shell via a 55 conductor cable. The random access projector interface (described in detail below) is connected directly to the computer's serial port.

The display and response system components are controlled by procedures and functions that have been incorporated into a Pascal library unit called APOLLO. APOLLO is written entirely in Pascal; it contains no assembly-language routines. APOLLO makes no use of, nor does it modify, the MS-DOS interrupt system; it is machine-independent.

\section{Timing}

Timing accuracy is $1 \mathrm{msec}$, which is sufficient for virtually any experiment carried out in the domain of perception or cognition. The display and the response systems make use of the same timing system, which operates as follows.

Hardware. The Intel 8253 counter/timer chip in the QuaTech CTM-10 interface is programmed to keep time by counting 1,500 pulses from an on-board 1.5-MHz crystal oscillator. The resulting $1-\mathrm{msec}$ pulses overflow to increment a 16-bit counting register that may be read as two consecutive bytes. The resulting data word equals the number of milliseconds since the last clock reset or rollover. The maximum value before rollover is 65,536 (i.e., $2^{16}$ ) msec, programmed in APOLLO to occur at $60,000 \mathrm{msec}=1 \mathrm{~min}$.

Software. In addition to clock initialization instructions contained in "InitBox," there are three timing routines. First, procedure "ResetClock" resets the timing register to 0 . Second, function "ElapsedTime" returns a longinteger value corresponding to the time, in milliseconds, since the last "ResetClock" call. "ElapsedTime" is central to all other timing functions in the system. Because it returns a long integer (32 bits), "ElapsedTime" will continue to increment for approximately 49.7 days before recycling. (In practice, because "ResetClock" is always called at the beginning of an experimental session, it never recycles.) The procedure "Wait (delay)" waits for delay milliseconds and replaces the less accurate Turbo Pascal Delay procedure. Finally, the "WaitUntil (sometime)" procedure delays program execution until a particular time after the last "ResetClock."

\section{Standard Projectors}

In addition to the four standard projectors, there is hardware and software to allow a fifth projector to be substituted for the random access one. Advancing and reversing of all standard projectors is accomplished under program control.

Hardware. The projectors are advanced by a $200-\mathrm{msec}$ optotriac "solid state relay" (Opto22 OAC5) closure, and reversed by a 500 -msec closure. Relay durations are under software control.

Software. The APOLLO procedure "ProjFor (projnum)" advances Projector "projnum" (projnum $=1-4$ ) by one carousel slot. Similarly, procedure "ProjRev (projnum)" reverses Projector projnum by one slot.

\section{Random Access Projector}

A Kodak random access projector is equipped with a motor that rotates the tray to any desired tray slot $(0-80)$. Ordinarily, a random access projector is controlled by a manual controller - that is, an operator dials in the desired slot and presses a button to execute the tray movement. This operation is under program control. Because random access projectors are quite costly and difficult to maintain, we have included only one of them in our projection system.

Hardware. The random access projector is interfaced via a MAST Corporation model 140-RS, which accepts a 300-baud BCD input from the computer's serial output port. The numbers input to the MAST range from 0-80 (they send the projector to slots 0-80), plus 99 and 98 , which turn the projector on and off, respectively.

Software. The APOLLO procedure to control the random access projector is "RaProj (slotnum)," where "slot- 
num" is an integer variable, restricted to the values $0-80$, 98 , and 99.

\section{Sound}

In many experiments, it is desirable to have a computergenerated tone operating in close conjunction with the display. For example, a tone may be used to signal the next display, or it might be used to cue the location of the stimulus, as in the Sperling (1960) partial report paradigm. The standard IBM PC tone generator is incorporated into the system software in a manner to be described below. The tone frequency in hertz is determined by the value of the APOLLO global integer variable, "Hertz."

\section{Accessing the Shutters and Tone}

Gerbrands tachistoscopic shutters are attached to each of the five projectors. (We built our own shutter drivers to save money and space.)

Hardware. The power supply that drives each shutter is designed to open the shutter with a $60-\mathrm{V}$ pulse and then instantly ramp down to $2.75 \mathrm{~V}$. The high pulse opens the shutter quickly; the low maintaining voltage, which is just sufficient to keep the shutter open, has the advantages of preventing overheating and allowing rapid closure, since the (spring-loaded) shutter closes in only a few tenths of a volt drop. A reverse-biased shunt diode is attached at the shutter end of the cable to reduce EMI at closure. The electrical schematic of the shutter driver is shown in Figure 4.

Software. The shutters can be turned on and off in any combination and in any sequence. Conceptually, the computer tone is considered to be a "sixth shutter." Thus, at any given time, the system is conceptualized as being in any of 64 (i.e., $2^{6}$ ) states, corresponding to each of the five shutters (open or closed), plus the tone (on or off). For ease of discourse, these six on/off components, five shutters plus the tone, will be collectively referred to as "shutters." Two APOLLO procedures are used to control the shutters: "Shutters (code)" is used for simple operations, and "Sequence" is used for complicated shutter operations and/or when very precise shutter timing is needed.

Procedure "Shutters (code)" effects a particular open/closed combination of the six shutters. The specific combination is determined by the value of the argument, "code." When "code" is in the $0-63$ range, it is conceptualized as a six-bit binary number with the lowestorder through the highest-order bits corresponding to the intended states of Shutters 1-5 and the tone, respectively. Within the six-bit number, 0 signals "closed" (or "off" in the case of the tone), and 1 signals "open" (or "on" in the case of the tone). So, for example, issuing the bi-

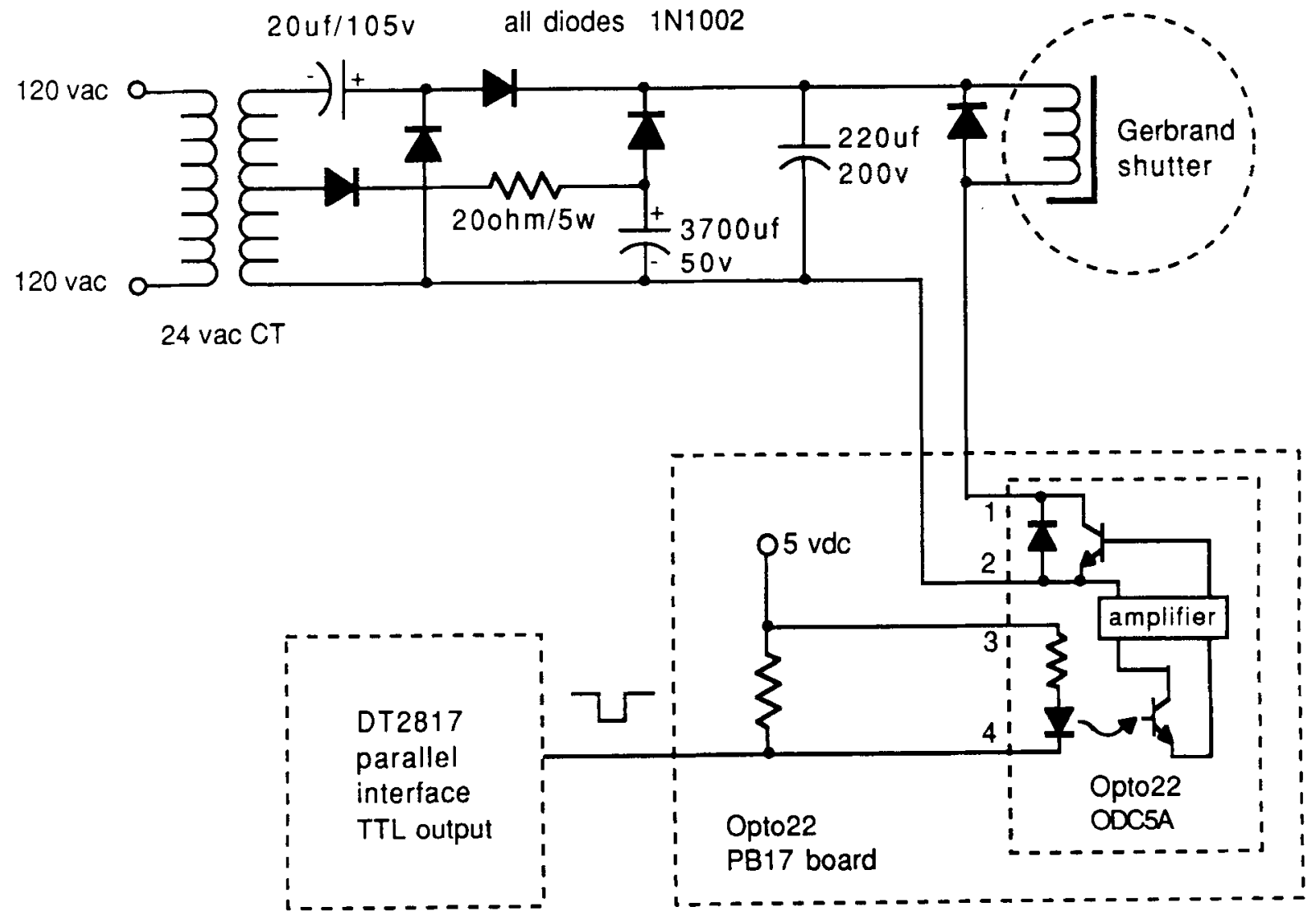

Figure 4. Schematic of shutter driver interface. The 200 resistor from the center tap of the transformer may be decreased in value to increase the holding voltage of the circuit. 
nary code 101010 would cause the shutters on Projectors 2 and 4 to be open, the shutters on Projectors 1, 3, and 5 to be closed, and the tone to be on (at frequency "Hertz"). (This code would be issued in the Pascal program with the instruction "Shutters (42)," since 42 is the decimal equivalent of 101010) ${ }^{1}$.

Procedure "Sequence" also allows the five shutters to be opened and closed, in conjunction with the tone, in any combination, but for precise times and in an arbitrarily complex sequence. The exact operation of "Sequence" will be described more fully in a later section.

\section{Luminance Attenuation}

Control over stimulus luminance is desirable in most experiments (e.g., when one wishes a relatively dim fix- ation point) and essential in some (e.g., when stimulus luminance is an independent variable). Most perception laboratories use either filter wheels or luminance wedges to control luminance. Bulky filter wheels would not fit in our display system; the wide beam emitted by the slide projectors precluded the use of luminance wedges. Thus, we devised a new device.

Hardware. Each projector is equipped with the luminance attenuation device schematized in Figures 5 and 6. This device, called a "flipper," incorporates four 2in. square neutral-density filters of $0.2,0.4,0.8$, and 1.5 $\log$ units attenuation, arranged in series with the least dense filter closest to the projector. The four filters are normally in the "down" state, out of the projector beam. Any combination of them can be flipped to the "up" state,

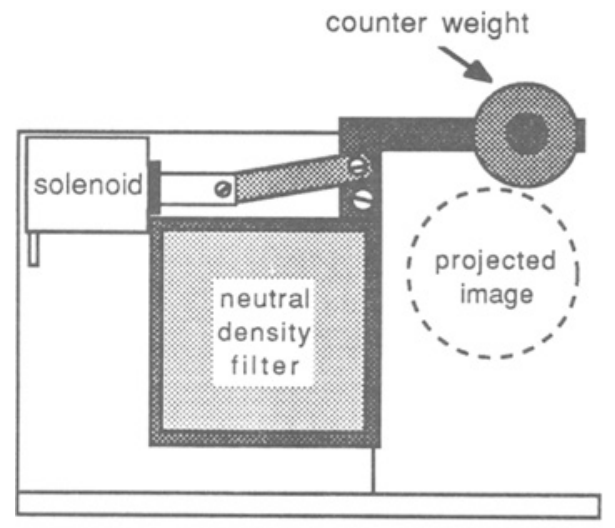

filter down

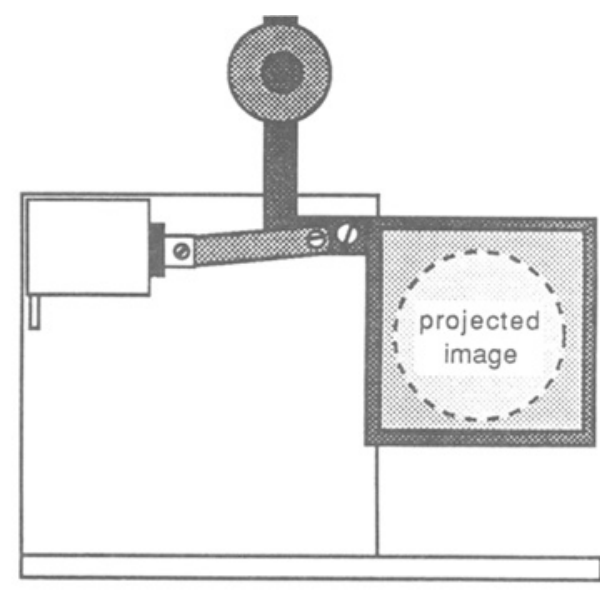

filter up

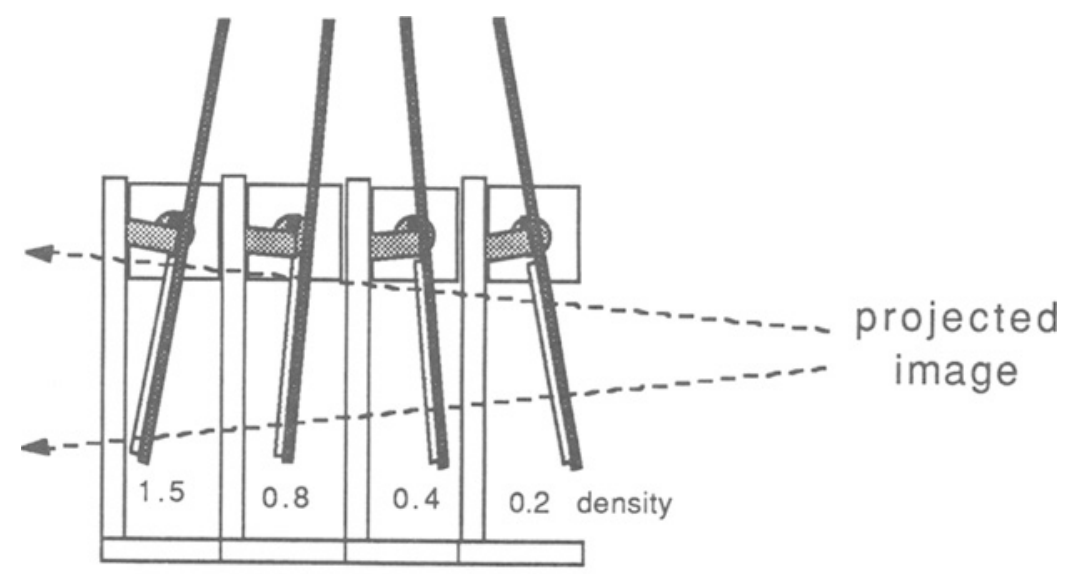

$43 / 4$ in $122 \mathrm{~mm}$

Figure 5. Schematic of luminance attenuation device (flipper). 


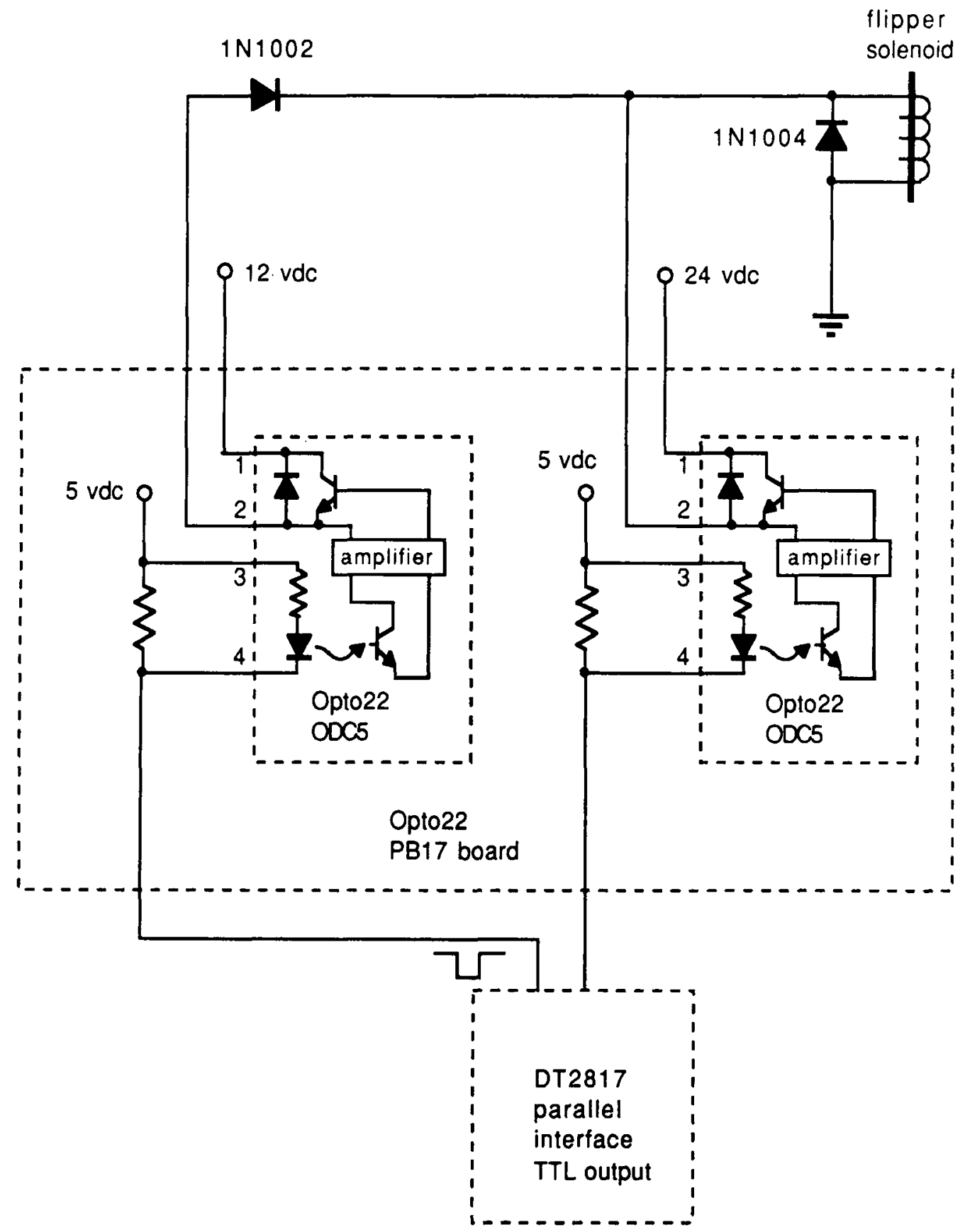

Figure 6. Schematic of driver interface for the flippers. Note that the circuit shown has both an 11-V and a 28-V driver. There must be one $28-\mathrm{V}$ driver for each solenoid, but only one $11-\mathrm{V}$ driver is needed to run several solemoids in holding position; $11 \mathrm{~V}$ can hold filters up once lifted but this does not provide adequate force to lift a filter, because the solenoids are rated for $24 \mathrm{~V}$.

in which they intersect the beam and attenuate the luminance. The 16 combinations of the four filters up/down define luminance attenuations ranging from 0 to $2.9 \mathrm{log}$ units in 16 , roughly equal steps. A period of approximately $1 \mathrm{sec}$ is required for the filters to change state. The metal-film filters are tilted $7^{\circ}$ to $10^{\circ}$ with respect to each other, to reduce interactive reflection that would pass filtered light back through the filters.
Each flipper is actuated by a Guardian 24-V solenoid. When first activated, the desired filters are flipped to the "up" state with a 28-V pulse from an open-frame linear power supply ( $24 \mathrm{~V}$ adjusted up $4 \mathrm{~V}$ ), which is replaced after 1 sec by a sustaining $11 \mathrm{~V}$ from a second linear supply ( $12 \mathrm{~V}$ adjusted down $1 \mathrm{~V}$; see Figure 7$)$. The latter voltage is sufficient to keep the filters up without overheating the light-duty solenoids. 
Software. The APOLLO procedure to control the flippers is "Flippers (projnum, atten)," where "projnum" is the projector number and "atten" is the desired degree of attenuation (a real variable ranging from 0.0-2.9). "Flipper" rounds to the nearest available optical density and then issues the appropriate solenoid activation code to the DT2817 parallel interface.

\section{Response Boxes}

Hardware. The response system consists of eight custom-built 16-key pads, each capable of sending an independent 4-bit response to the computer along with a strobe and inverted strobe signal at each keypress (Figure 7). Responses can be untimed (ended with a pseudo-"enter" key) or timed (in which case a response consists of only a single keypress). Because the response boxes themselves latch the most recent keypress and present that information continually on the parallel input lines of the DT2817s, there must be a way to identify the repetition of a particular key that would not change the data on the lines. The necessary handshaking is provided by custom interface card (Figure 8) plugged into the QuaTech PBX-721 expansion card that receives and latches the keypress strobe signal for each response box. For every keyboard in the system a pair of Schmitt trigger IC gates receives both the strobe and the invert strobe signals, inverts one signal, and routes both to an AND gate that triggers a clock-edged latch. The latches can be read and reset by the PPI chip in the expansion card. The dual strobe signals are very effective in eliminating electrical noise, since both must invert simultaneously to opposite logic states for a keypress to be registered.

Software. Two APOLLO routines, "KeyTimer" and "KeyTyper," are used to access the response boxes. "KeyTimer" is used when responses are to be timed, and "KeyTyper" is used when response time is not relevant. Three eight-element arrays (one element corresponding to each box), globally defined in APOLLO, are relevant to response-box software. "Online" is a Boolean array, indicating which boxes are in use by subjects during an experimental session. "KeyTimes" is a real array into which reaction times are placed by "KeyTimer." "KeyVals" is an integer array into which the value of the response is placed by either "KeyTimer" or "KeyTyper."

Both "KeyTimer" and "KeyTyper" operate by continually polling the response latches on the interface card and the activated response boxes until all active boxes have responded. "KeyTimer" allows only a single-key response, which is recorded in "KeyVals," and whose time since the issuance of the "KeyTimer" instruction is recorded in "KeyTimes." "KeyTyper," in contrast, accepts the " $F$ " key as an indication that the response has been completed; subjects are instructed to treat the

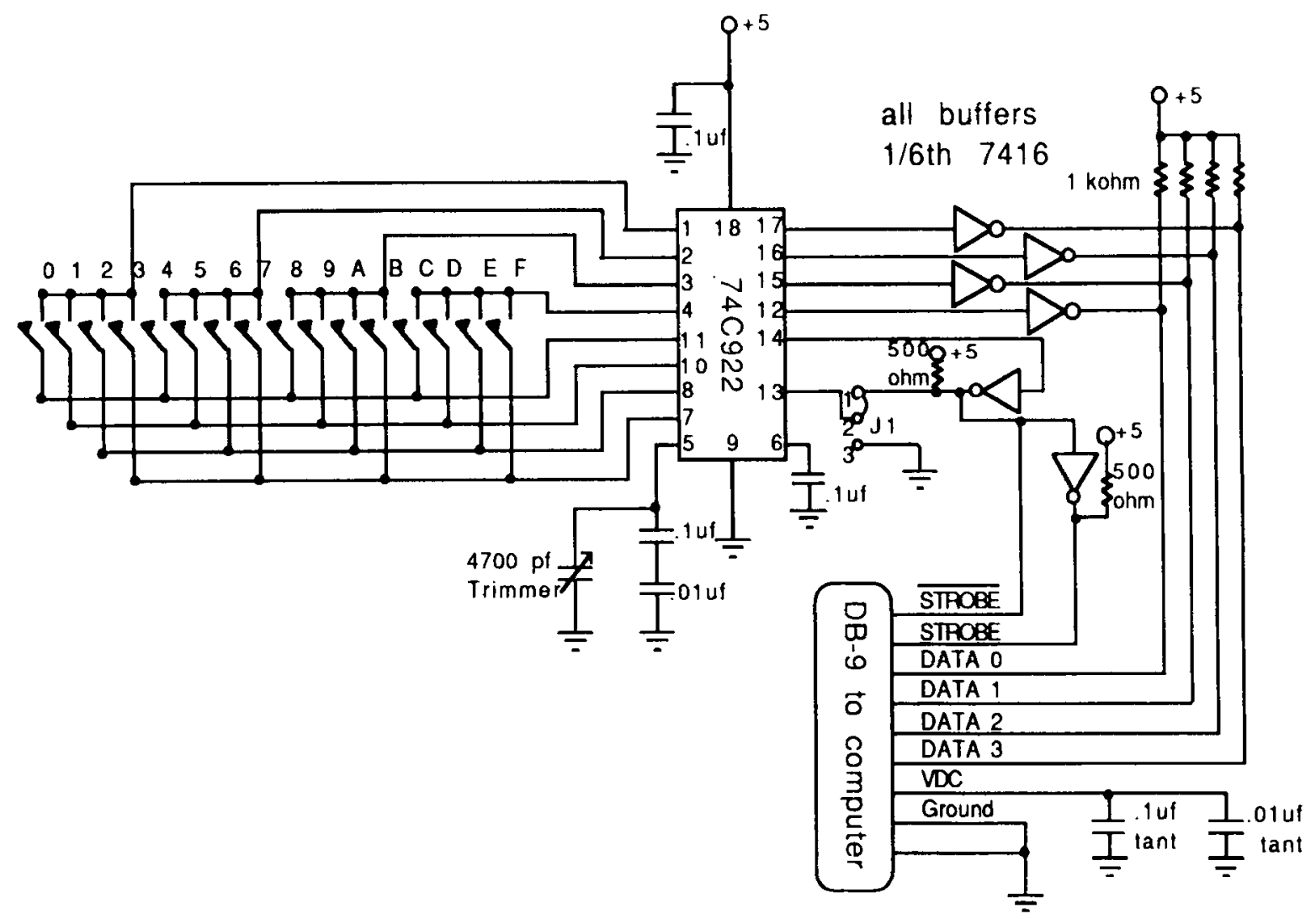

Figure 7. Schematic of the 16-key response boxes. With jumper $\mathrm{J1}$ in the configuration shown, each keypress latches that key's data onto the data lines until the next keypress. With $\mathrm{J} 1$ in the alternate configuration, the data lasts only the duration of the keypress. 


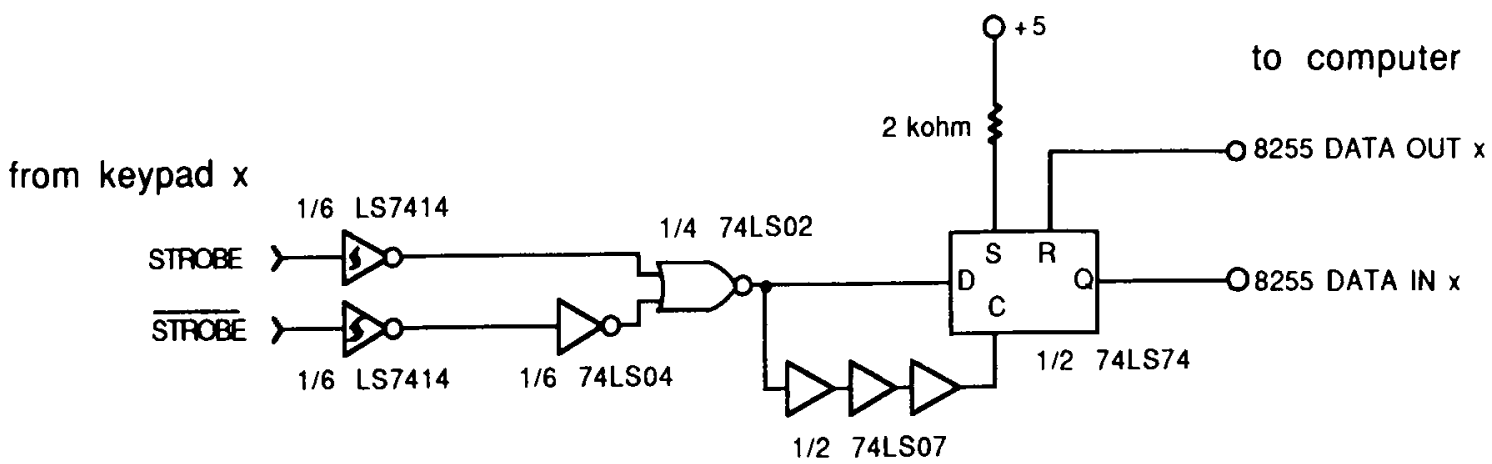

Figure 8. Schematic of keyboard strobe interface built onto a QuaTech prototyping module that plugs into the QuaTech PBX-721 expansion card. This diagram represents a circuit for one response box. Eight such circuits reside on the card in our system.

"F" key like an "enter" or "return" key. (The "F" key is in approximately the same keyboard position as a return key.) "KeyTyper" accepts a response that is (arbitrarily) limited to four digits, which it records in "KeyVals." "KeyTyper" also allows error correction: if the subject wishes to change a response prior to typing the " $F$ " key, the subject can do so by typing the " $E$ " key, which erases the entry in "KeyVals."

\section{HOW "SEQUENCE" WORKS}

"Sequence" is the central display routine. In this section we provide an overview of "Sequence" and its variables in APOLLO.

\section{Overview of "Sequence"}

"Sequence" is used for executing complex, precisely timed sequences of stimulus displays. The basic idea is that a series of cumulative times are assigned to successive locations of an APOLLO-defined integer array called "Seqtimes." The codes corresponding to events that are to occur at these times are assigned to corresponding locations of an APOLLO-defined integer array called "seqevents." (Recall that a code that ranges from 0-63 determines the configuration of the five shutters and the tone.) When "Sequence" is called, the events specified in "seqevents" are executed at the times specified in "seqtimes." An APOLLO-defined variable, "seqptr," points to the event number (that is, the "seqtimes/seqevents" location) being executed at any given time. Of special importance is the ability to escape from "Sequence" temporarily, and return to the main program to perform housekeeping chores such as changing projectors, updating conditions, getting responses, etc.

\section{An Example Using "Sequence"}

Suppose part of an experiment consists of a series of 40 trials. On each trial, a sequence of seven events (numbered 0-6) occurs, as is described in Table 1A, column 2. Column 3 lists the event codes that correspond to the desired events. Finally, column 4 lists the Pascal code responsible for filling "seqtimes" and "seqevents." By convention, "seqtimes" and "seqevents" are filled in a procedure called "Fillseq."

Cumulative times starting at trial onset. Time 0 is defined to be trial onset. At time 0 , a masking slide (in Projector 3) comes on, and it remains on for $1,500 \mathrm{msec}$. A fixation point (in Projector 1) along with a warning tone occurs during the last 500 msec of the mask-that is, starting at Time 1,000. At Time 1,500, the shutters close, the tone turns off, and all is dark and quiet and peaceful for $500 \mathrm{msec}$. Starting at Time 2,000 , a target picture is shown from Projector 5 for a variable duration, "targettime"' (that ranges, say, from 50 to $200 \mathrm{msec}$ ). Following target offset is a dark, variable-duration interval, "isi" (which ranges, perhaps, from 0 to $300 \mathrm{msec}$ ), after which the mask reappears for $300 \mathrm{msec}$. The stimulus-onset asynchrony (SOA) from the start of Trial $n$ to the start of Trial $n+1$ is to be $5,000 \mathrm{msec}$. During the relatively long interval between the offset of the final mask and the beginning of the next trial, the target projector must be changed, and other housekeeping activity, such as choosing new values of "targettime" and "isi," must be carried out.

Calling "Fillseq" and "Sequence" from the main program. Table 1B illustrates how "Fillseq" and "Sequence" are called from the main Pascal user program. Sometime prior to beginning the 40 trials, the tone frequency is set to $1000 \mathrm{~Hz}$. The trials then begin. At the beginning of each trial, housekeeping chores for that trial are performed; these include computing the appropriate carousel slot for the target-slide projector (Projector 5), computing appropriate values of "targettime" and "isi," and sending Projector 5 to the appropriate slot (which is done using "RaProj," since Projector 5 happens to be the random access projector). Following the housekeeping, "Fillseq" is called, which, as indicated in Table 1A, sets up the appropriate events to occur at the appropriate times. Finally, to initiate the display, "seqptr" is reset to 0 (to begin the display at Event 0 ) and "Sequence" is executed.

When "Sequence" is initially called (on Trial 1), it begins executing the display immediately. The last thing that happens in "Sequence" (Event 6) is that "seqtimes [6]" 
Table 1

Pascal Code Demonstrating the Use of Sequence

\section{A. Desired trial events and the Fillseq procedure}

\begin{tabular}{|c|c|c|c|}
\hline Event & $\begin{array}{l}\text { State of System } \\
\text { (Projecters on) }\end{array}$ & $\begin{array}{l}\text { Event Code } \\
\text { (Binary) }\end{array}$ & Pascal Instructions for Fillseg \\
\hline 0 & Mask (Proj 3) & 000100 & $\begin{array}{l}\text { Seqtimes }[0]:=0 \\
\text { Seqevents }[0]:=\mathrm{p} 3\end{array}$ \\
\hline 1 & $\begin{array}{l}\text { Mask, Fixation, } \\
\text { tone (Proj } 1,3 \text { ) }\end{array}$ & 100101 & $\begin{array}{l}\text { Seqtimes }[1]:=1000 \\
\text { Seqevents }[1]:=p 1+p 3+\text { lone }\end{array}$ \\
\hline 2 & Everything off & 000000 & $\begin{array}{l}\text { Seqtimes }[2]:=1500 \\
\text { Seqevents }[2]:=0\end{array}$ \\
\hline 3 & $\begin{array}{l}\text { Target (Proj 5) } \\
\text { for targettime ms }\end{array}$ & 010000 & $\begin{array}{l}\text { Seqtimes }[3]:=2000 \\
\text { Seqevents }[3]:=\mathrm{p5}\end{array}$ \\
\hline 4 & $\begin{array}{l}\text { Everything off } \\
\text { for isi ms }\end{array}$ & 000000 & $\begin{array}{l}\text { Sequimes }[4]:=2000+\text { targettime; } \\
\text { Seqevents }[4]:=0 ;\end{array}$ \\
\hline 5 & Mask (Proj 3) & 000100 & $\begin{array}{l}\text { Seqtimes }[5]:=2000+\text { targettime+isi; } \\
\text { Seqevents }[5]:=p 3\end{array}$ \\
\hline 6 & $\begin{array}{l}\text { Everything off } \\
\text { (escape to program) }\end{array}$ & 000000 & $\begin{array}{l}\text { Seqtimes }[6]:=5000 \\
\text { Seqevents }[6]:=\text { escape; }\end{array}$ \\
\hline
\end{tabular}

\section{B. The main program from which Fillseq and Sequence are called}

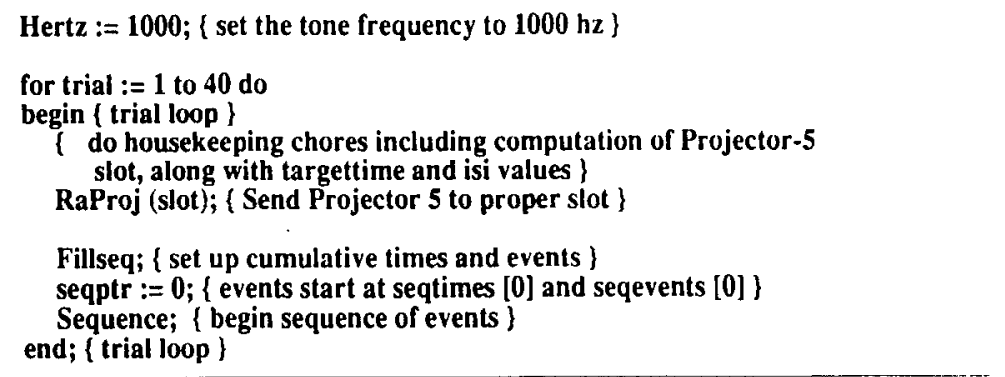

is set for $5,000 \mathrm{msec}$. The special "seqevents" code, "escape" (which happens to be decimal 99) causes an immediate exit from "Sequence," back to the main program; however, "Sequence" will "remember" that the next event is supposed to occur $5,000 \mathrm{msec}$ hence. At that point, the housekeeping chores will be performed anew, "Fillseq" recalled, "seqptr" reset to 0, and "Sequence" recalled. On all trials subsequent to the first, "Sequence" will start by waiting until the previously set interval $(5,000 \mathrm{msec})$ has elapsed before it begins executing the display events. In this manner, precise control over trialto-trial SOA can be achieved, while still using the times between displays (or any relatively long time during which no new display event occurs) for housekeeping.

\section{An Alternative to "Sequence"}

If Sequence seems unnecessarily complicated, we offer a simpler alternative. "WaitUntil (SomeTime)" delays program execution until "SomeTime" in milliseconds since the last "ResetClock." Events to be executed at particular times, such as shutters, tones, flippers, or projector advance, are specified by single line commands separated by "WaitUntil" commands. Commands that involve a delay such as projector advance and flipper control interfere with long-range timing if simple "Wait" statements are used without regard for subroutine execution time. "WaitUntil" does not delay execution if the designated time has elapsed during another procedure, and thus long-range timing integrity is preserved.

\section{SYSTEM LIMITATIONS}

The system is designed expressly for experiments that use 35-mm slides as stimuli. This use of $35-\mathrm{mm}$ slides in a computer-based laboratory has advantages and disadvantages that are described in detail by Loftus (1982). The major limitations of the present laboratory follow.

\section{Timing Limitations}

Individual shutters cannot reopen for about $50 \mathrm{msec}$ after closing, because the shutter drivers are capacitive and take time to recharge. Timing becomes inaccurate below about $5 \mathrm{msec}$, because of mechanical inertia.

The standard carousel projectors require roughly $1 \mathrm{sec}$ to advance. This limitation takes two forms. First, as it is currently programmed, the software must "hang" in 
the procedure "ProjFor" for the 200-msec period necessary to issue the advance signal (just as one must press a projector advance button for at least $200 \mathrm{msec}$ for the projector to advance) ${ }^{2}$. Suspension of program execution could be eliminated with interrupt-driven timing routines at a considerable cost in program complexity, with hardwired timing circuitry at a cost in electrical noise immunity, or with a dynamic scheduler (under consideration in our lab). Second, and more problematic, the projector requires about $1 \mathrm{sec}$ to physically advance. This places a limit on how rapidly long sequences of visual stimuli can be shown (as in the sorts of paradigms used, for example, by Intraub, 1980, 1984; Neisser \& Beller, 1965; Potter, 1976; or Sperling, Budiansky, Spivak, \& Johnson, 1971). If one cycles continuously through all five projectors, the minimum target-target SOA is approximately $250 \mathrm{msec}$. In contrast, Intraub (e.g., 1980) reports experiments in which movie film has been used to present an indefinitely long series of pictures at $111-\mathrm{msec}$ SOAs.

Finally, the flipper filters require about $1 \mathrm{sec}$ to change state, because small solenoids were used to facilitate tight spacing of filters. This means that one cannot easily manipulate luminance within a rapid stimulus-presentation sequence.

Luminance limitations. Luminance is precisely controlled within a given experimental session by using neutral-density filters. However, the slide-projector bulbs undergo slight luminance change over their lifetimes. This property imposes the major limit on the accuracy of luminance control.

\section{Running Experiments}

Time required. A typical picture-memory experiment might involve 20 groups of 5-8 subjects per group. The total time required for such an experiment is approximately $25 \mathrm{~h}$, broken down as follows:

(1) It takes $4 \mathrm{~h}$ to modify and debug previous Pascal programs. Three programs are used for a given experiment: one to randomize presentation orders, one to run the experiment, and one to compute means and standard deviations from the raw data (and convert the raw data into a form suitable for statistical analysis).

(2) Twenty hours are required to run the subjects. Data, collected via the response boxes, are analyzed at the end of each experimental session, and presented to subjects during debriefing.

(3) An hour at the end of the experiment for statistical analysis.

Experimental paradigms. Examples of experimental paradigms that have been or can be used are the following:

(1) In a variety of visual-memory paradigms, we have examined acquisition of information from the iconic image, picture-luminance effects, picture-priming effects, and perceptual and conceptual masking effects. Performance measures, all obtainable from the response boxes, include forced-choice and old/new recognition, number of picture details recalled, confidence in subsequent recog- nition, and Sperling (1960) partial report of digit stimuli (Loftus, 1985a, 1985b; Loftus et al., 1985).

(2) Response-contingent stimulus presentation procedures have been used in several ways. The first is to investigate subjective duration of the iconic image, using a synchrony-judgment task (see Efron, 1970). In this paradigm, a target picture is displayed, followed by a variable-length blank interval, followed by a signal, such as a visual mask, or an auditory beep. The subject's task is to adjust the length of the interval, so that the posttarget signal seems to coincide with the target's complete disappearance. This is accomplished over a series of trials, by increasing or decreasing the interval's duration in accord with the subject's report of the temporal relation between signal and icon disappearance.

The second procedure is a variation on a temporal integration paradigm (Di Lollo, 1980; Eriksen \& Collins, 1966), in which two halves of a stimulus display are presented in sequence, and some aspect of the perceptual completeness of the display (such as a completeness rating) is measured.

(3) Psychophysical brightness judgment tasks (see Stevens, 1957) have been used to assess effects of stimulus complexity on perceived brightness.

\section{Replicability}

How easily can the laboratory that we have described here be replicated elsewhere? Our system software (the APOLLO unit) is transportable. System hardware is less transportable, and the ease of replication depends on the complexity required.

Software replicability. Although the software is currently implemented on an IBM-compatible computer, it incorporates, as noted earlier, no assembly language routines, or use of the MS-DOS interrupt system. Moreover, it is written entirely in Pascal; thus, it is transportable to other computer systems with little modification. The major required change in a non-MS-DOS environment would be to write new basic timing functions ("ResetClock," "Ticker," "ElapsedTime") suitable for the available timing hardware. Given a correctly working "ElapsedTime," the rest of the software system would follow with minor changes.

Hardware replicability. Much of the system hardware, including all projectors and shutters, the computer itself, the timing interface, the $\mathrm{I} / \mathrm{O}$ cards, the random access projector interface, and the solid-state relays for standard projector advance/reverse, may be bought off the shelf; it is thus easy to replicate. However, the response boxes, the flippers, the shutter drivers, and the soundproof, temperature-controlled display system housing are all custom made and are, of course, less easy to replicate. To build a complete, exact replication of the laboratory hardware would cost approximately $\$ 10,000$ and would require approximately $1,200 \mathrm{~h}$ on the part of a competent technician. A bare-bones version of the current system (such as two standard slide projectors with shutters, no 
software-controlled luminance control, one 3-key response box, and no display system housing) has been built for approximately $\$ 3,200$; it took approximately $20 \mathrm{~h}$.

An up-to-date version of our APOLLO software may be obtained by sending a formatted disk (5.25-in. $360 \mathrm{~K}$ or $1 \mathrm{MB}$, or 3.5-in. $720 \mathrm{~K}$ ) and return addressed mailer to the second author.

\section{REFERENCES}

DiLoLLo, V. (1980). Temporal integration in visual memory. Journal of Experimental Psychology: General, 109, 75-97.

EFron, R. (1970). The relationship between the duration of a stimulus and the duration of a perception. Neuropsychologia, 8, 37-55.

ERIKSEN, C. W., \& Collins, J. F. (1966). Some temporal characteristics of visual pattern perception. Journal of Experimental Psychology, 74, 476-484.

INTRAUB, H. (1980). Presentation rate and the representation of briefly glimpsed pictures in memory. Journal of Experimental Psychology: Human Learning \& Memory, 6, 1-12.

INTRAUB, H. (1984). Conceptual masking: The effects of subsequent visual events on memory for pictures. Joumal of Experimental Psychology: Learning, Memory \& Cognition, 10, 115-125.

LofTus, G. R. (1982). Picture memory methodology. In K. Rayner (Ed.), Handbook of research methods in human memory and cognition (pp. 257-285). New York: Academic Press.

LorTus, G. R. (1985a). On worthwhile icons: Replies to DiLollo and Haber. Journal of Experimental Psychology: Human Perception \& Performance, 11, 384-388.

LofTus, G. R. (1985b). Picture perception: Effects of luminance level on available information and information-extraction rate. Journal of Experimental Psychology: General, 114, 342-356.

Loftus, G. R., Giluispie, S., Tigre, R. A., \& Nelson, W. W. (1984). An Apple II-based slide-projector laboratory. Behavior Research Methods, Instrumentation, \& Computers, 16, 447-453.

LoFTUS, G. R., \& GINN, M. (1984). Perceptual and conceptual processing of pictures. Journal of Experimental Psychology: Learning, Memory, \& Cognition, 10, 435-441.

Loftus, G. R., HANnA, A., \& LeSter, L. (1988). Conceptual masking: How one picture steals attention from another picture. Cognitive Psychology, 20, 237-282.

LofTUs, G. R., \& HoGDEN, J. (1988). Picture perception: Information extraction and phenomenological persistence. In G. H. Bower (Ed.), The psychology of leaming and motivation, Vol. 22. New York: Academic Press.
Lortus, G. R., Johnson, C. A., \& Shimamura, A. P. (1985). How much is an icon worth? Journal of Experimental Psychology: Human Perception \& Performance, 11, 1-13.

Loftus, G. R., Truax, P. E., a Nelson, W. W. (1986). Age-related differences in visual information processing: Quantitative or qualitative? In C. Schooler \& K. W. Schaie (Eds.), Cognitive functioning and social structures over the life course (pp. 59-78). Norwood, NJ: Ablex.

Neisser, U., \& Beller, H. K. (1965). Searching through word lists. British Journal of Psychology, 56, 349-358.

PotTer, M. C. (1976). Short-term conceptual memory for pictures. Journal of Experimental Psychology: Human Learning \& Memory, 2, 509-522.

PotTer, M. C., \& Levy, E. I. (1969). Recognition memory for a rapid sequence of pictures. Journal of Experimental Psychology, 81, 10-15.

ReINITZ, M. (1987). The effects of semantic priming on visual encoding. Unpublished doctoral dissertation, University of Washington, Seattle.

SPERLING, G. (1960). The information available in brief visual presentations. Psychological Monographs, 74, 1-29.

Sperling, G., Budiansky, J., Spivak, J., Johnson, M. C. (1971). Extremely rapid visual search: The maximum rate of scanning letters for the presence of a numeral. Science, 174, 307-311.

Stevens, S. S. (1957). On the psychophysical law. Psychological Review, 64, 153-181.

\section{NOTES}

1. To facilitiate programming, there are global constants defined in APOLLO called "p1," "p2," "p3," "p4," "p5," and "tone," which are set to $1,2,4,8,16$, and 32 , respectively. This allows both the code corresponding to any combination of shutters and the tone to be expressed as the sum of the relevant components. In the example given, the instruction to open the shutters on Projectors 2 and 4, along with the tone, would be "Shutters (p2+p4+tone)."

2. "ProjRev" must hang for 500 msec. Also, the timing delays in both "ProjFor" and "ProjRev" could be implemented in hardware with LM555 timer ICs driving the Opto22 ADC5 relay modules. These oneshots, however, are notorious for spontaneously activating in response to background electrical noise. We are currently programming a dynamic scheduler to eliminate program suspension during projector advance and filter changes.

(Manuscript received July 2, 1988; revision accepted for publication October 21, 1988.) 